\title{
Robust Feed-Forward/Feedback Design for Tape Transport
}

\author{
Dirk Tenne ${ }^{1}$ and Tarunraj Singh ${ }^{2}$ \\ Department of Mechanical \&5 Aerospace Engineering, \\ State University of New York at Buffalo \\ http://code.eng.buffalo.edu
}

\begin{abstract}
The purview of this paper is the design of controller which include knowledge of parametric uncertainties and their distributions. The parameter distributions are approximated by a finite set of points which are calculated by the unscented transformation. This set of points are used to design robust controllers which minimize the worst performance of the plant over the domain of uncertainty. The proposed technique is illustrated on a tape transport system. Two approaches to arrive at robust prefilters and feedback controllers are proposed. The first which involved sequential design of the feedback and prefilter and the second which simultaneously arrive at optimal parameters of the feedback gain matrix and the time-delay prefilter. Numerical results illustrate desirable response over the operating region of the system.
\end{abstract}

\section{Introduction}

This paper presents the development of a robust controller for high-speed and low tension tape transport systems, which are controlled by the supply voltages to the motor amplifiers for each reel. Current models of the tape drive have been presented by Panda, Lu and Engelmann [1,2], and Mathur and Messner [3]. The tape drive exhibits slowly varying dynamics as the reel packs are changing. In addition, the tape stiffness undergoes fluctuations due to air entrapment between tape layers. These varying characteristics are modeled as parameter uncertainties. The design of a feedback controller consisting of a state feedback and integral control part has been investigated to achieve the desired speed and tension requirements. Panda [1] developed a LQR feedback control. Mathur and Messner [3] noted a large sensitivity to parameter changes of state feedback controllers, and designed a controller using the sequential loop closing approach. This work presents a robust feedback controller design similar to the minimax technique. The proposed robust design

\footnotetext{
${ }^{1}$ Graduate Student, Control, Dynamics and Estimation Laboratory, University at Buffalo

${ }^{2}$ Associate Professor, Control, Dynamics and Estimation Laboratory, University at Buffalo
}

minimizes the statistics of a cost function and significantly reduces the computational requirements exploiting the unscented transformation developed by Julier and Uhlman [4], which approximate statistics of processes. Furthermore, a robust feed-forward controller has been designed to reduce the startup time of the tape and to cancel the oscillations at the end of the desired startup time. Two concepts for designing the feed-forward/feedback control have been investigated. The first described as the sequential approach selects the feedback gain based on a statistical LQR cost. Subsequently, the feed-forward controller is designed to minimize a metric of the tracking error. The second approach has been posed as an optimization problem which minimizes a combined cost function including the LQR and tracking error metrics, to arrive at the concurrent controller.

Robust control laws describe a set of control parameters which despite varying plant characteristics yield reasonable control performance. A controller designed for the nominal plant can perform considerably poorly when the plant characteristics change. Assuming prescribed knowledge of the plant variations, the robust control design identifies the worst operating point of a plant and seeks the optimal set of control parameters for this plant. This trade-off compromises the controller's performance at the nominal plant with the benefit of satisfactory performance over the anticipated plant variations.

The plant variations are characterized in the mathematical model by a set of parameters, for example the eigenfrequency of a mechanical model can be prescribed to lie in an interval with lower and upper bounds. Doyle [5], Bryson and Mills [6] defined the parameter deviation interval $\mathcal{P}$, as a box centered at the nominal plant, where the worst parameter combination has been approximated to lie at the corners of $\mathcal{P}$ (which is true for conservative systems [7]). In real systems additional information about the uncertainty of the mathematical model may be available which can be incorporated into the design process. This research investigates robust control design including uncertain model parameter, which can be described by their probabilistic distribution, i.e. the probability density function (pdf).

This paper opens with a discussion on encapsulat- 
Table 1: Central moments of uniform distribution

\begin{tabular}{|cc|}
\hline \multicolumn{2}{|c|}{ central moments } \\
\hline$m_{p}$ & $\sigma_{p}^{2}$ \\
\hline \hline$\frac{p_{u b}+p_{l b}}{2}$ & $\frac{\left(p_{u b}-p_{l b}\right)^{2}}{12}$ \\
\hline
\end{tabular}

ing the distribution of parametric uncertainties using the unscented transformation [4]. This is followed by the conception of a cost function, which results in controllers emulating the minimax controller. The next section illustrates the sequential and concurrent controller design for the tape transport system.

\section{Statistical Robust Controller Design}

\subsection{Model Parameter Distributions and Ap- proximations}

Consider the uncertain parameter vector $p$, which is bounded in the interval space

$$
\mathcal{P}: p \in\left[\begin{array}{ll}
p_{l b} & p_{u b}
\end{array}\right] .
$$

The lower $\left(p_{l b}\right)$ and upper $\left(p_{u b}\right)$ limits on the parameters are known quantities, which for example represent mechanical limits such as the stiffness coefficient of a spring. Additional information about the probabilistic distribution of the parameter vector $p$ is often available, which can be described by the joint probability density function

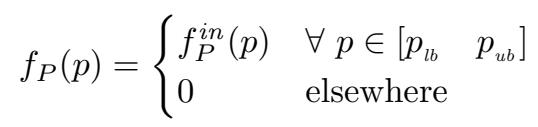

where $f_{P}^{i n}(p)$ is the pdf within the parameter interval.

Assuming that the variations of the plant parameters are independent of each other, it is sufficient to describe one dimensional distributions since the joint pdf is composed of the product of the independent pdf's. If no further knowledge about the parameter variations is available, i.e. every possible plant realization is equally likely, a uniform distribution should be considered, where $f_{P}^{i n}(p)=1 /\left(p_{u b}-p_{l b}\right)$. The statistical robust controller approximates the probabilistic distribution by a finite number of central moments. Table 1 shows the first two moments of the uniform distributed parameter, which can be obtained by integrating the pdf of equation 2. The first two central moments are called the mean $\left(m_{p}\right)$ and the variance $\left(\sigma_{p}^{2}\right)$, which are measures of the expected value and its spread over the parameter probability space.

\subsection{Unscented Transformation}

The unscented transformation is used in this work to describe the probabilistic characteristics of a cost function subject to parameter uncertainties. Consider a nonlinear transformation of the random variable $X$ with mean $\bar{x}$ and variance $P_{x}$

$$
Y=g(X)
$$

where we would like to approximate the statistics (eg. mean and variance) of the transformed random variable $Y$.

The key feature of the unscented transformation is that it approximates the probability distribution rather than approximating the nonlinear function by a Taylor series. The unscented transformation selects a $\sigma$-set consisting of $2 n+1$ points, which are perturbations from the mean by a scaled deviation. The deviations $\sigma_{i}$ are defined as the columns of the matrix square root of $P_{x}[4]$

$$
\sigma= \pm \sqrt{P_{x}} .
$$

The $\sigma$-set is defined as:

$$
\begin{aligned}
\zeta_{0} & =\bar{x} \\
\zeta_{i} & =\zeta_{0}+\sqrt{(n+\kappa)} \sigma_{i} \text { for } i=1 \ldots 2 n,
\end{aligned}
$$

such that the $\sigma$-set exhibits the same probabilistic characteristics as the random variable $X$ and $\kappa$ is a design variable of the unscented filter.

The $\sigma$ set is transformed by the nonlinear relationship

$$
\eta_{i}=g\left(\zeta_{i}\right) \quad \text { for } \quad i=1 \ldots 2 n
$$

and the estimate of the mean and variance of $Y$ is obtained by the weighting scheme:

$$
\begin{aligned}
\bar{\eta} & =\sum_{i=0}^{2 n} w_{i} \eta_{i} \\
P_{\eta} & =\sum_{i=0}^{2 n} w_{i}\left(\eta_{i}-\bar{\eta}\right)\left(\eta_{i}-\bar{\eta}\right)^{T}
\end{aligned}
$$

where

$$
w_{o}=\frac{\kappa}{n+\kappa}, \quad w_{i}=\frac{1}{2(n+\kappa)}
$$

has been selected to match the first four central moments of $X$.

\subsection{Statistical Quadratic Performance Index}

Optimal control seeks a control input to a dynamic system, which minimizes a certain performance criteria. For LQG controller, for example, the performance index is a quadratic integral cost and for time-delay filter the cost is composed of the energy remaining in the plant. However, to achieve a satisfactory performance over the entire parameter space as discussed in the previous section, the performance index should be evaluated at the worst combination of all permissible plant parameters. Doyle [5] proposed a mini-max cost:

$$
\min _{u} \max _{p \in \mathcal{P}} J(x, p, u)
$$


whose solution $u$, minimizes the worst performance over the uncertain parameter space $\mathcal{P}$. The simultaneous maximization and minimization requires the identification of the worst parameter combination during the minimization operation, which can be considerably simplified by prescribing a set of plant parameters approximating the worst case. El Ghaoui and Bryson [7] have identified that the maxima appear at the corners of the parameter space $\mathcal{P}$ for conservative systems and near the corners for lightly damped systems.

To ensure that the minimax approach does not eliminate uncertain plant models which might lie at locations other than the corners of the uncertain hypercube, it is necessary to perform an exhaustive search of the uncertain domain. This can be computationally prohibitive. Using the statistics of the cost function over the uncertain parameter space provides an efficient approach for the determination of a robust controller. The relationship between the parameter statistics and the performance index statistics is obtained via the unscented transformation (UT) developed originally for nonlinear filtering by Julier and Uhlmann [4].

In contrast to the minimax approach (equation 11) the statistical cost considers the moments of the quadratic performance index [8]:

$$
\min _{u} \operatorname{mom}_{f_{P}(p)} J(x, p, u) .
$$

The robustness is achieved by requiring the mean and the deviation of $J(x, p, u)$ to be minimized. Minimizing the mean $m_{J}$ endeavors to yield the best performance and minimizing the deviation $\sigma_{J}$ corresponds to reducing the dispersion, which results in robustness. The resulting cost is:

$$
\operatorname{mom}_{f_{P}(p)} J(x, p, u)=m_{J}+\sigma_{J} .
$$

\section{Tape Transport Control}

The tape drive consists of two reels which supply and file the tape according to the direction of transport. The tape transport dynamics can be modeled by a nonlinear model [3], where the voltages applied to the motor $\left(u_{1}, u_{2}\right)$ represent the inputs and the tape tension $(T)$ and the tape speed at the read-write head $\left(v_{r w}\right)$ are the outputs. The nonlinearities are introduced by the changing inertia of the reels as the tape is transported from the supply to the file reel. With respect to the time constants of the tape transport dynamics, the variation of the inertias can be neglected. Furthermore, neglecting the terms including the tape thickness, we arrive at a reduced order linear state-space model [3]:

$$
\begin{aligned}
\dot{x} & =A x+B u \\
y & =C x,
\end{aligned}
$$

where the state is composed of the tape tension and the angular velocities of reels one and two. The matrices
$A, B$ and $C$ are taken from [3] and repeated here for convenience.

$$
\begin{gathered}
A=\left[\begin{array}{ccc}
-D\left(\frac{r_{1}^{2}}{J_{1}}+\frac{r_{2}^{2}}{J_{2}}\right) & D \frac{\beta r_{1}}{J_{1}}-K r_{1} & K r_{2}-D \frac{\beta r_{2}}{J_{2}} \\
\frac{r_{1}}{J_{1}} & -\frac{\beta}{J_{1}} & 0 \\
-\frac{r_{2}}{J_{2}} & 0 & -\frac{\beta}{J_{2}}
\end{array}\right] \\
B=\left[\begin{array}{cc}
-D K_{t} r_{1} / J_{1} & D K_{t} r_{2} / J_{2} \\
K_{t} / J_{1} & 0 \\
0 & K_{t} / J_{2}
\end{array}\right] \\
C=\left[\begin{array}{ccc}
0 & r_{1} / 2 & r_{2} / 2 \\
1 & 0 & 0
\end{array}\right]
\end{gathered}
$$

The tape stiffness and damping are denoted by $K$ and $D$, and the reel radii and inertia are represented as $r$ and $J$. The motor torque constant is $K_{t}$ and the viscous friction coefficient is denoted by $\beta$.

The time varying characteristics of the reel inertia and tape stiffness are modeled as parametric uncertainties which are bounded in the interval space $\mathcal{P}$. These plant variations can result in undesirable tape transport characteristics, and feedback controllers based on pole placement are very sensitive to these modeling errors [3]. The nominal plant parameters are considered to be the average radius and the nominal tape stiffness

$$
p_{\text {nom }}=[15.5 \mathrm{~mm} \quad 2010 \mathrm{~N} / \mathrm{m}]
$$

The upper and lower bound of the radius are the maximum reel radius $\left(r_{\max }=21.2 \mathrm{~mm}\right)$ and the inner radius of the reel $\left(r_{i}=9.75 \mathrm{~mm}\right)$ respectively. The stiffness variations are assumed to be in the range of $\pm 10 \%$ of the nominal value.

The tape transport is controlled by the voltages applied to the motor amplifiers, where the design parameters are the tape speed and the tension in the tape. The requirements on the high-speed and low-tension tape transport are taken from Mathur and Messner [3], where experiments have been carried out with a tape speed of $5 \mathrm{~m} / \mathrm{s}$ and a tape tension of $0.28 \mathrm{~N}$. A startup time of $150 \mathrm{~ms}$ has been demonstrated.

\subsection{Sequential Controller Design}

To achieve the desired speed and tension, a statistically robust feedback controller in an error space has been designed. Furthermore, a robust feedforward controller has been designed to cancel the oscillations at the end of the desired startup time. The following text describes the design of the robust feedback controller based on the Linear Quadratic Regulator (LQR) cost. Subsequently, the robust time-delay filter is designed based on the suppression of the oscillations at the final maneuver time.

The state equation 14 is augmented by the error dynamics:

$$
\dot{x}_{e}=C x-r,
$$

which together with the control law

$$
u=-k_{1} x-k_{2} x_{e}
$$




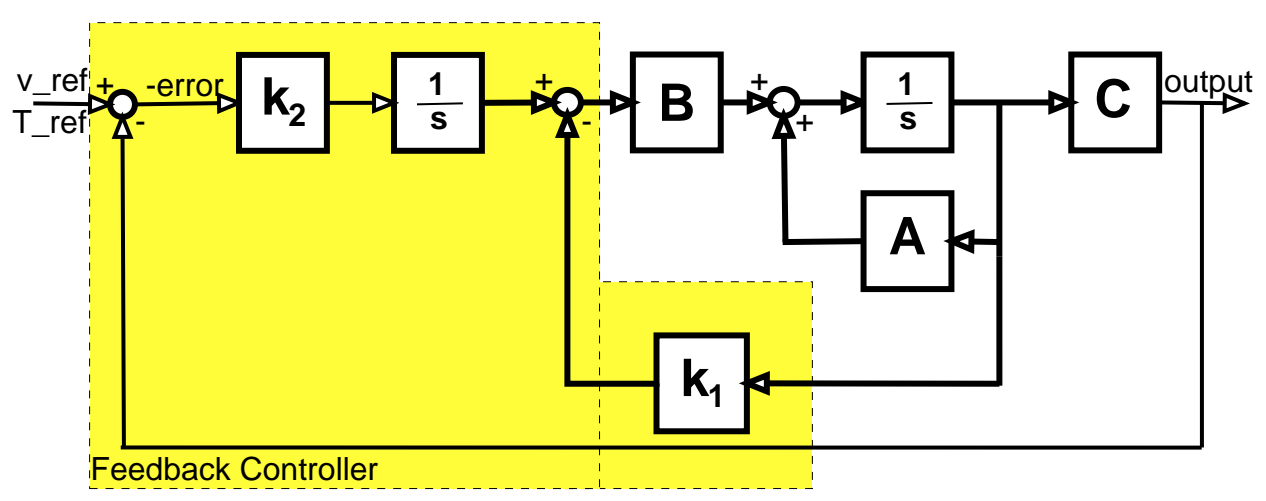

Figure 1: Block diagram of the Feedback controller

ensures that the system output tracks the reference signal $r=\left[v_{\text {ref }} T_{\text {ref }}\right]^{T}$. Defining the augmented state by $z=\left[\begin{array}{ll}x & x_{e}\end{array}\right]^{T}$ the state space model as shown in the block diagram (Figure 1) can be constructed:

$$
\dot{z}=\tilde{A} z+\left[\begin{array}{c}
0 \\
-I
\end{array}\right] r,
$$

where the closed loop system matrix is given by:

$$
\tilde{A}=\left[\begin{array}{cc}
A-B k_{1} & -B k_{2} \\
C & 0
\end{array}\right]
$$

The LQR cost can be calculated by first solving the steady state Lyapunov equation:

$$
P \tilde{A}+\tilde{A}^{T}=-\tilde{Q},
$$

where $\tilde{Q}=Q+K^{T} R K$ is the augmented weighting matrix consisting of the state weighting matrix $Q$ and the control weighting matrix $R$. The feedback gain matrix $K$ is composed of the state feedback and error feedback gains $K=\left[\begin{array}{ll}k_{1} & k_{2}\end{array}\right]$. The LQR cost depends on the initial condition:

$$
J_{\mathrm{LQR}}=z(0)^{T} P z(0),
$$

where

$$
z(0)=\tilde{A}^{-1}\left[\begin{array}{l}
0 \\
I
\end{array}\right] r
$$

The optimal feedback gain based on the nominal plant

$$
\begin{aligned}
k_{1 \mathrm{LQR}} & =\left[\begin{array}{ccc}
-21.7445 & 0.3638 & -0.0512 \\
21.7445 & -0.0512 & 0.3638
\end{array}\right] \\
k_{2 \mathrm{LQR}} & =\left[\begin{array}{cc}
22.3607 & -0.2236 \\
22.3607 & 0.2236
\end{array}\right]
\end{aligned}
$$

can be derived by solving the algebraic Riccati equation.

As discussed in the aforementioned text the LQR feedback controller is optimal if the tape drive is operating in its nominal range and it provides a minimal degree of robustness subject to parameter perturbations. The plant parameter perturbations are assumed to be uniformly distributed over the parameter deviation interval such that the probability density function constitutes a hypercube of height $h=1 /(0.2 K \Delta r)$, where $\Delta r=r_{\max }-r_{i}$ is the interval length of the reel radius. The unscented transformation will be used to approximate the first moments of the parameter space. The $\sigma$-set creates a set of plant realizations with the corresponding set of performance indices, where the mean resembles the average cost and the variance measures the spread over the parameter deviation. By minimizing the statistics of the cost function equation 24, we arrive at the statistical robust feedback controller with the feedback gain of:

$$
\begin{aligned}
k_{1 \text { unscented }} & =\left[\begin{array}{rrr}
-63.1015 & 0.0268 & 0.0508 \\
0.2020 & 0.0142 & 0.0154
\end{array}\right](27 \mathrm{a}) \\
k_{2 \text { unscented }} & =\left[\begin{array}{ll}
157.0169 & -88.8470 \\
187.0247 & 106.9412
\end{array}\right] \quad(27 \mathrm{~b})
\end{aligned}
$$

The minimization has been performed using the optimization toolbox of MATLAB. Due to the manner in which the Lyapunov function represents the cost of unstable systems, a new cost function had to be conceived of which in essence is the same as the original cost:

$$
J_{\mathrm{LQR}}^{*}=-\frac{1}{J_{\mathrm{LQR}}} .
$$

Subsequent to the design of the robust feedback controller, the feedforward controller is developed to create finite-time response of the closed loop system. This design is based on the time-delay/input-shaper approach proposed by Singh and Vadali [9]. The multiple input shaper configuration is shown in Figure 2 including the amplitudes and time delays, which are selected to cancel the oscillations at the final switch time. This input shaper is designed using the nominal tape parameters. To achieve parameter robustness, the input shaper parameters are selected based on the statistical performance evaluation. The performance index

$$
E=\left(z-z_{\mathrm{des}}\right)^{T} M\left(z-z_{\mathrm{des}}\right),
$$

has been selected as a quadratic cost function consisting of pseudo energy terms ensuring that the desired 


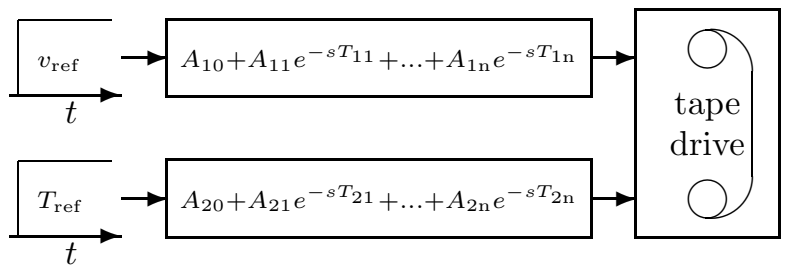

Figure 2: Multi-input time-delay shaper block diagram

Table 2: Input Shaper Parameters of the sequential and concurrent design, (switching times in ms, amplitudes in $\%$ of 1 )

\begin{tabular}{|c|c||c|c||c|c||c|c|}
\hline \multicolumn{4}{|c||}{ Sequential } & \multicolumn{4}{c|}{ Concurrent } \\
\hline \multicolumn{2}{|c||}{ Velocity } & \multicolumn{2}{|c||}{ Tension } & \multicolumn{2}{|c||}{ Velocity } & \multicolumn{2}{c|}{ Tension } \\
\hline \hline$T_{i}$ & $A_{i}$ & $T_{i}$ & $A_{i}$ & $T_{i}$ & $A_{i}$ & $T_{i}$ & $A_{i}$ \\
\hline- & - & 0 & 53.9 & 0 & 74.2 & 0 & 43.7 \\
\hline 0 & 98.9 & 32 & 31.7 & 2 & 5.4 & 6 & -21.0 \\
\hline 22 & 5.4 & 55 & 13.9 & 33 & 4.5 & 8 & -15.8 \\
\hline 23 & 3.7 & 80 & 3.9 & 53 & 16.4 & 13 & -3.2 \\
\hline 103 & -2.0 & 86 & 1.0 & 83 & -12.7 & 21 & 138 \\
\hline 113 & -5.6 & 98 & -7.9 & 86 & -1.2 & 37 & 4.2 \\
\hline 129 & -2.1 & 112 & -5.7 & 89 & 2.8 & 83 & -9.8 \\
\hline 141 & 1.6 & 141 & 9.3 & 93 & 10.7 & 93 & -36.3 \\
\hline
\end{tabular}

states are reached, where $M$ is a positive definite matrix and the desired states $z_{\text {des }}$ are calculated based on equation 25. The deterministic minimax optimization procedure proposed by Singh [10] minimizes the worst performance over the parameter interval. It involves an exhaustive search calculating the residual energy over the parameter deviation interval, whereas the statistical approach evaluates the cost at carefully chosen plant variations. By minimizing the statistics of the cost function (equation 29)

$$
\min _{T_{i}, A_{i}} \operatorname{mom}_{r_{1}, K} E
$$

we arrive at the robust time-delay shaper as shown in Table 2. A shaper with seven time delays has been selected to suppress the oscillations. The left column of Table 2 shows the switch times and the amplitudes of the sequential design for the two reference inputs. Note that the values have been rounded and the switching times are measured in milliseconds (ms) and the amplitudes are displayed in percent ( $\%$ of 1 ).

The robustness of the sequential controller design is demonstrated by testing various tape drive realizations. In particular, the nominal system is described by both reels with the same radius, the startup system is described by a full supply reel and empty file reel, and the end system is described as the other extreme. Simulations have been carried out, which accelerate the tensionless tape from zero velocity up to the reference speed of $5 \mathrm{~m} / \mathrm{s}$. During the acceleration of the tape, the tension is required to reach the reference tension and
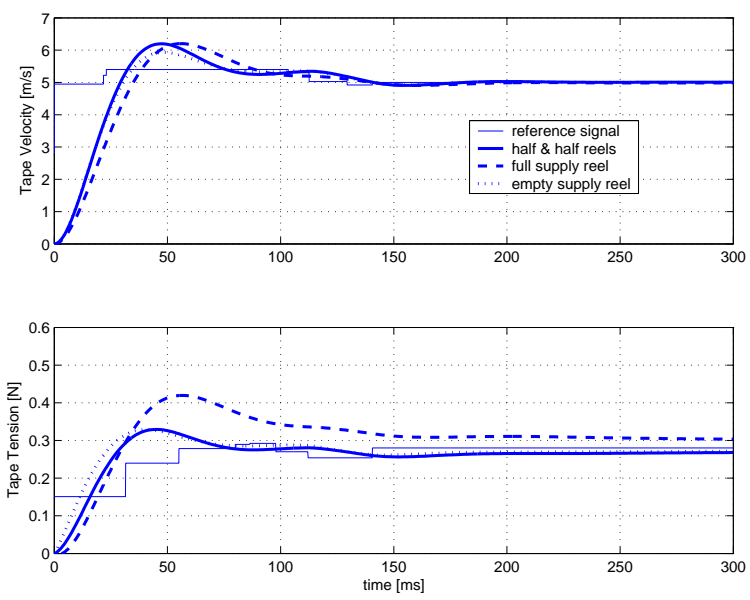

Figure 3: Tape acceleration for three tape drive realizations with sequential controller

the peak should not exceed the tape's yield stress. Figure 3 illustrates the tape acceleration process for the three reel settings. The tape stiffness has been kept constant at the nominal stiffness. The tape dynamics are reasonable robust as the reel radii are changing. It can be noted that the reference inputs are reached in about $150 \mathrm{~ms}$, whereas significant overshoot of the tape tension occurred.

\subsection{Concurrent Controller Design}

The performance of the sequentially designed controller can be improved by performing a concurrent design of the feedback part and the input shaper. The concurrent design is motivated by Muenchhof [11] and its robustness is achieved by minimizing the statistical cost of equation 12. The approximation of the moments of the cost function significantly reduces the computational burden as compared to the "brute-force" minimax approach. The optimization is performed on the combined cost function of the LQR-cost and the pseudo energy:

$$
J_{\text {concurrent }}=\omega J_{\mathrm{LQR}}^{*}+\omega E+(1-2 \omega) t_{f}
$$

where the final switch time $t_{f}$ has been included. The significance of the time can be adjusted by the weighting parameter $\omega$. The following results have been generated by using a weight of $\omega=0.46$. Table 2 shows the input shaper parameters of the concurrent design on the right column. The inclusion of the final switch time in the cost function reduced the input shaper duration from $141 \mathrm{~ms}$ to $93 \mathrm{~ms}$. The integrator feedback gain and the state feedback gain are:

$$
\begin{aligned}
& k_{1 \text { concurrent }}=\left[\begin{array}{ccc}
-21.9970 & 0.0184 & 0.0160 \\
-1.6794 & 0.0179 & 0.0102
\end{array}\right] \\
& k_{2 \text { concurrent }}=\left[\begin{array}{ll}
157.0452 & -88.8510 \\
187.2541 & 106.9460
\end{array}\right] .
\end{aligned}
$$



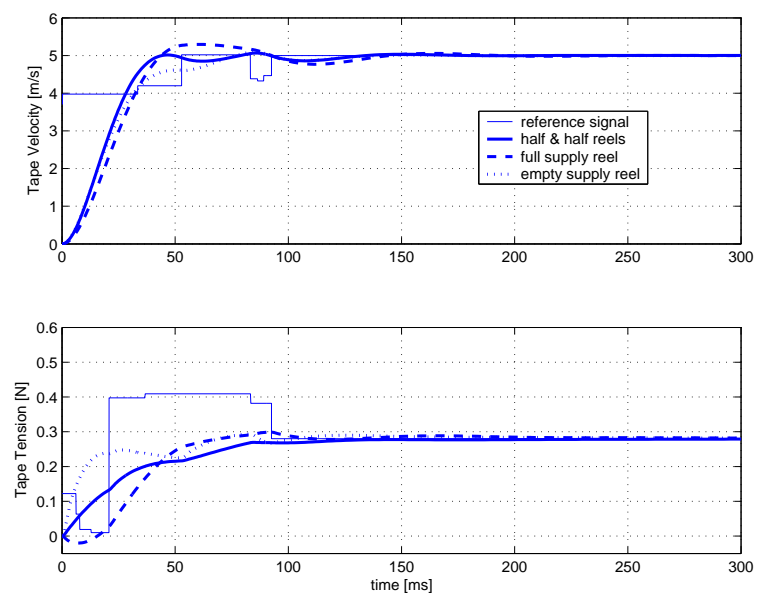

Figure 4: Tape acceleration for three tape drive realizations with concurrent controller

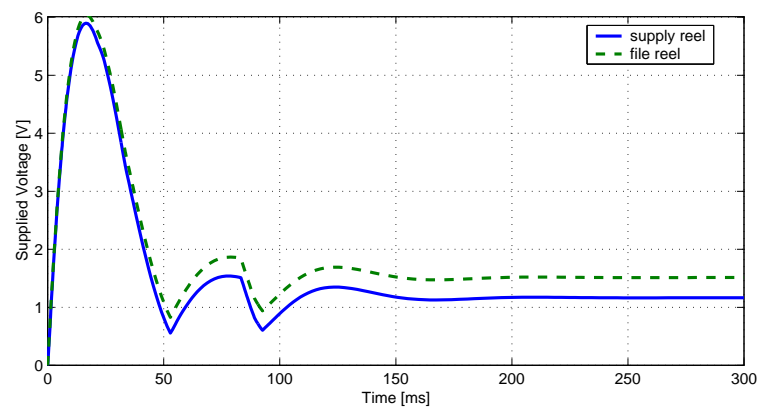

Figure 5: Control input voltages of the reel motors by using the concurrent controller

Figure 4 illustrates the tape dynamics for the three tape drive realizations. The overshoot in the tape tension has been significantly reduced and the time to reach the reference inputs has been reduced to about $100 \mathrm{~ms}$. The control inputs of the reel motors are shown in Figure 5. The peak voltage occurs at $17 \mathrm{~ms}$ of about 6 Volts, which demonstrates realistic control efforts.

\section{Conclusion}

The major contribution of this work is a new technique for the design of controllers robust to modeling uncertainties. The domain of uncertainty is assumed to be known as is the distribution of the parameters. The unscented transformation is exploited to approximate the distribution of the cost function as a function of the uncertain plant parameters. This leads to a problem formulation which requires a small number of plant realizations to design controllers which closely emulate minimax controllers designed using exhaustive search of the parameter space. The tape drive mechanism which is characterized by time varying reel inertias and tape stiffness is used to illustrate the proposed technique. A sequential and concurrent design is car- ried out and the performance of the concurrent design is shown to robustly control the system over its entire range of operation, while achieving excellent transient behaviour.

\section{Acknowledgments}

The authors would like to thank Dr. S.P. Panda for his valuable inputs.

\section{References}

[1] Shiba P. Panda and Andrew P. Engelmann. Modeling and control system design of reel-to-reel tape drives. In ACC, Anchorage, Alaska, May 2002.

[2] Shiba P. Panda and Yan Lu. Tutorial on control systems design in tape drives. In $A C C$, Denver, Colorado, June 2003.

[3] Priyadarshee D. Mathur and William C. Messner. Controller development for a prototype high-speed lowtension tape transport. IEEE Trans. on Cont. Systems Tech., 6(4):534-542, July 1998.

[4] Simon J. Julier, Jeffrey K. Uhlmann, and Hugh F. Durrant-Whyte. New approach for filtering nonlinear systems. In $A C C$, volume 3 , pages $1628-$ 1632, 1995.

[5] J. C. Doyle. Analysis of feedback systems with structured uncertainty. In IEE Proceedings part D, volume 129(6), pages 242-250, 1982.

[6] Arthur E. Bryson and Raymond A. Mills. Linearquadratic-gaussian controllers with specified parameter robustness. J. of Guid., Control and Dyn., 21(1):11-18, Feb. 1998.

[7] L.E. El Ghaoui and Arthur E. Bryson. Worst case parameter changes for stabilized conservative systems. In Proc. of the AIAA Conf. on Guid., Navig. and Cont., New Orleans, LA, Aug. 1991.

[8] Dirk Tenne and Tarunraj Singh. Efficient minimax control design for prescribed parameter uncertainty. Submitted for review to the. J. of Guid., Control and Dyn.

[9] Tarunraj Singh and S. R. Vadali. Robust timedelay control. ASME J. of Dyn. Systems, Measurements, and Cont., 115:303-306, 1993.

[10] Tarunraj Singh. Minimax design of robust controllers for flexible systems. J. of Guid., Control and Dyn., 25(5):868-875, 2002.

[11] M. Münchhof and T. Singh. Concurrent feedforward/feed-back design for flexible structures. In AIAA Guid., Navig. and Cont. Conf., Monterey, California, Aug. 2002. 